\title{
Revisión de las contrataciones en el sector público
}

\author{
Jorge Arturo Yangales Vega \\ jyangales@ hotmail.com \\ Universidad Cesar Vallejo \\ Lima - Perú
}

\section{RESUMEN}

OBJETIVO: Este artículo de revisión tuvo como propósito inicial destacar la influencia de las contrataciones del Estado en el desarrollo del país, así como su intervención en el presupuesto de la entidad, gasto público, las condicionantes en los aspectos de obligaciones sociales y medio ambientales. METODOLOGIA: La metodología utilizada para este artículo ha sido el enfoque cuantitativo, además con la revisión y el análisis de una variedad de datos bibliográficos de organismos, tanto de nivel nacional como internacionales, los cuales guardan relación con el tema. RESULTADOS: Asimismo, como resultado se obtuvo la existencia de un beneficio enlazado a las contrataciones con el estado, siendo esta el aportar a la potenciación del país a través de las inversiones y el consumo. CONCLUSIÓN: Se concluyó que las contrataciones del Estado resultan beneficiosas en gran medida para el potenciamiento del país por las diversas inversiones que son realizadas, como también al alto consumo estatal del mercado interno, pero que actualmente presenta elevados índices de corrupción.

Palabras clave: contrataciones; estado; entidades; gasto; público. 


\title{
Contracts in the public sector
}

\begin{abstract}
OBJECTIVE: This review article had the initial purpose of highlighting the influence of State contracting in the development of the country, as well as its intervention in the entity's budget, public spending, the conditioning factors in the aspects of social and environmental obligations. METHODOLOGY: The methodology used for this article has been the quantitative approach, in addition to the review and analysis of a variety of bibliographic data from organizations, both national and international, which are related to the subject. RESULTS: Likewise, as a result, the existence of a benefit linked to contracting with the state was obtained, this being the contribution to the empowerment of the country through investments and consumption. CONCLUSION: It was concluded that state contracting is beneficial to a great extent for the empowerment of the country due to the various investments that are made, as well as the high state consumption of the internal market, but that currently presents high levels of corruption.
\end{abstract}

Keywords: hiring; state; entities; spending; public.

Artículo recibido: 15 noviembre. 2021 Aceptado para publicación: 10 diciembre 2021

Correspondencia: jyangales@hotmail.com Conflictos de Interés: Ninguna que declarar 


\section{INTRODUCCIÓN}

Actualmente en diversos países se ha observado un incremento en la tasa de desconfianza por parte de la ciudadanía hacia el gobierno, ya que los índices de corrupción son exuberantes en diversos países y los delitos culposos aumentan, razón por la cual gran parte de los ciudadanos no desean pagar impuestos o peor aún, desconfían del gobierno de turno y las licitaciones o compras realizadas por el Estado. Ante esto Bonelli \& Cabral (2018), manifestaron que los lazos comprador- proveedor en los contratos públicos se basan en la búsqueda del beneficio propio y enriquecimiento ilícito, tal es el caso de 167 contratos entre empresas privadas y el Estado que fueron investigados por presentar indicios de fraude, pese a que las autoridades presentaban grados de instrucción y experiencia en los puestos públicos. Actualmente, los intereses privados demostraron contar con una influencia negativa en calidades que, desde la consideración pública y la liquidez altera negativamente los costos públicos, es decir, los gastos gubernamentales no son coherentes con la compra realizada por los organismos, dejando entrever que hay una mala praxis.

Ruiz \& Delgado (2020), aportaron que el proceso de contrataciones en las instituciones públicas se relaciona con la protección de los recursos públicos en el transcurso de las licitaciones. Es así que el autor determina que el control interno es llevado a cabo por la persona facultada a través de procedimientos, sistemas de información, seguimiento etc., con el fin de administrar eficazmente los recursos públicos, sin embargo, esto es omitido muchas veces porque las autoridades no cumplan su labor fiscalizadora (Rodriguez, 2020).

Para Rivas, et al. (2019), la contratación pública sobrepasa la idea de contrato administrativo y contrato de administración, siendo que la contratación pública aprecia de manera importante a la economía y al PBI nacional. Asimismo, el sistema de contratación pública repercute en el uso de los fondos estatales, considerándose como prioridad que dentro de la gestión pública se desarrolle eficazmente la efectuación de los contratos públicos pactados. Es por ello que los autores proponen adecuar la nueva tecnología en el sistema de contrataciones públicas, ya que se necesita emplear de manera segura, eficaz, transparente los procesos y, sobre todo, llevar a cabo contratos confiables para el desarrollo del país (Ramió, 2020). 
Pinto, et al. (2018), determinan que los contratos por parte de la administración pública, se establecen como una labor compleja desde una perspectiva de monitoreo e inspección en la fase de ejecución, los cuales permiten reconsiderar lo necesario para el potenciamiento y mantenimiento de la gestión pública, ya que sin una adecuada gestión no se podría hablar de eficiencia ni dinamismo, siendo esto un punto clave en adquisiciones del estado (Anibal \& Sano, 2018).

Gimeno \& Guerrero (2020), comentan que la pandemia causada por la COVID-19, ha remecido diversos sistemas sanitarios en el mundo dejando entrever la consistencia financiera de cada país, a su vez el autor proporciona sus consideraciones a la gestión pública y su modelo de contratación. Además, el autor recomienda realizar cambios en la adquisición de manera que se considere la calidad y la eficacia, además de potenciar la sostenibilidad económica del país, de manera que prime el desarrollo en base a una contratación pública que tenga en cuenta no solo a un sector de la población, sino a un al país en su conjunto (Peñate, 2020).

Gonzales (2017), considera que la regulación de las contrataciones se merman por el alto control y la rigidez, la cual daña la eficacia de las compras, por lo cual el gobierno indagó soluciones llevando a cabo la nueva gestión pública, la cual emplea la subasta inversa en donde los proveedores presentan ofertas, el proceso es el siguiente: primero un proveedor lanza la oferta, seguidamente se evalúa y compiten aquellos que presenten el costo más bajo, finalmente el estado decide y elige el que posea la licitación más baja; es así que la subasta electrónica permite agilizar los procesos de contratación pública permitiendo llevar de manera ordenada y veraz el sistema de control (Payne, 2017).

Espitia, et al. (2019), comentan que la contratación pública es el área que más impacto recibe con respecto a la corrupción, por tal motivo debería de recibir más atención a nivel naciones, regional y local. Como principales motivos se encuentra al gobierno de turno, el cual maneja un diseño institucional escaso con toma de decisiones malintencionadas; asimismo, otro factor importante es el ciudadano que ignora el impacto de daños en la economía pública, ya que desconoce los instrumentos necesarios para enfrentar tal mal. Es por ello, que los autores recomiendan aplicar reformas institucionales, así como enriquecer la ley y fomentar la participación ciudadana en la prevención de desvíos dinerarios públicos. 


\section{ESTRATEGIAS METODOLÓGICAS O MATERIALES Y MÉTODOS}

La metodología de la presente investigación se sustentó en la recolección de datos y contrastación de información pertinente, todo esto bajo una revisión sistemática de buscadores confiables. A su vez se utilizaron dos esquemas, el esquema de revisión y el esquema de PRISMA, los cuales brindan un orden y rigurosidad estricta respecto a las fuentes consultadas.

\section{Tipo y diseño de investigación}

La presente investigación estudió la variable "contrataciones en el sector público" y tuvo como objetivo principal despejar la duda acerca de la influencia que tienen las contrataciones del Estado en el desarrollo del país, así como su intervención en el presupuesto de la entidad, gasto público, las condicionantes en los aspectos de obligaciones sociales y medio ambientales. Es decir, la variable en mención ha sido estudiada bajo una relación causal o una influencia de una sobre la otra, esto con la finalidad de demostrar su relevancia en la realidad (Espinoza, 2018).

Se llevó a cabo mediante una revisión sistemática, la cual es un proceso que consisten en recolectar artículos para proporcionar una mirada amplia de un fenómeno en específico, de modo que con distintas fuentes se pueda elaborar un resumen cuidadoso y significativo que contribuya a conocer un tema. Este tipo de investigación resulta una manera excelente para recolectar y contrastar datos (Karunananthan, 2021).

El esquema de la revisión del artículo y su desarrollo se muestra a continuación:

\section{Su esquema se muestra a continuación:}

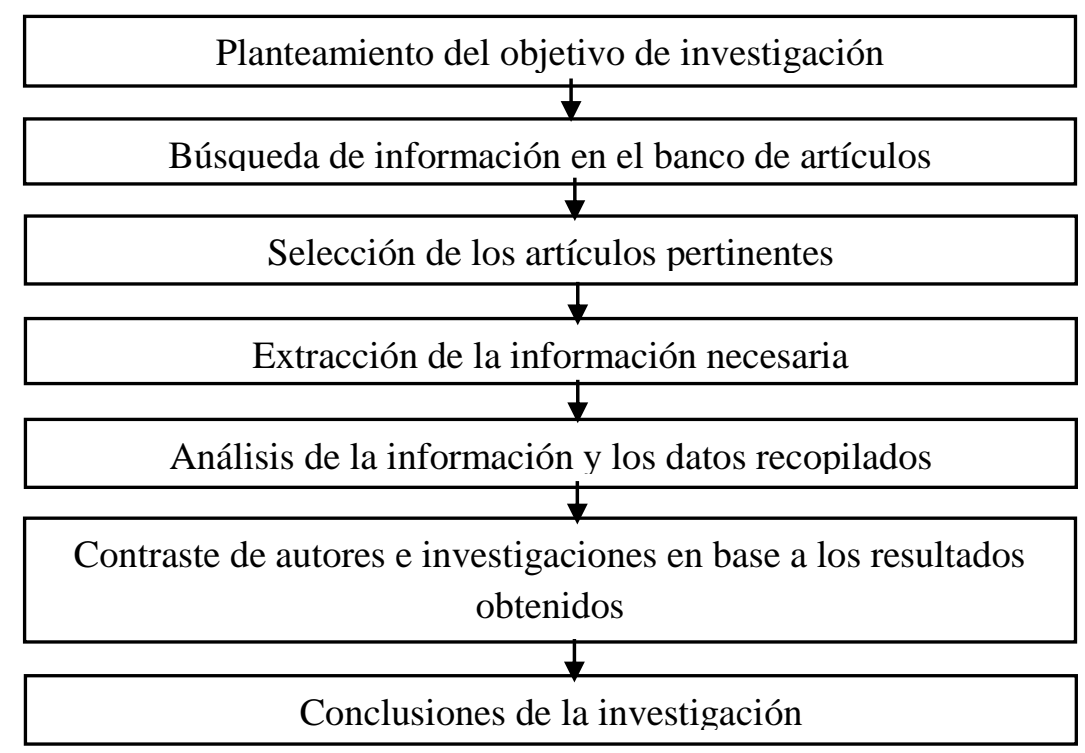

Figura: Esquema de la revisión del artículo 


\section{Técnicas e instrumentos de recolección de datos}

La presente investigación se realizó de forma eficiente porque se recolectó gran cantidad de artículos acerca de las contrataciones en el Sector Público, asimismo, la recopilación se basó en fuentes confiables como artículos, libros digitales y tesis, de manera que los datos fueron contrastados de diferentes autores y países.

El tratamiento de la investigación fue mediante una revisión sistemática documental científico, las cuales fueron seleccionadas por tener ciertas características similares, tales como:

- Artículos dentro del periodo 2017-2021.

- Artículos escritos en el idioma inglés (97\%), español (1\%), portugués (1.5\%) y otros $(0.5 \%)$.

- Selección de artículos en base al título de publicación, presencia de la variable de estudio.

- Selección de artículos por resultados y conclusiones similares a los de la investigación.

- Búsqueda de 2 tesis para el contraste de información.

- Selección de 2 libros digitales actuales para el contraste de información.

- Elección de revistas indexadas confiables.

- Variedad de autores y países.

\section{Procedimientos}

Los datos obtenidos forman parte de una revisión sistemática y bibliografía trasparente, los criterios para su inclusión fueron el año de publicación, el contenido pertinente en la investigación, y criterios como la realidad en las que se desarrollaban y los resultados que los investigadores obtuvieron durante su proceso.

Para la realización del estudio se utilizaron buscadores de internet tales como Scielo, Scopus, Google Académico, Ciencia Latina y repositorios internacionales confiables, para la selección de los mismos se utilizaron las palabras clave como "contrataciones",

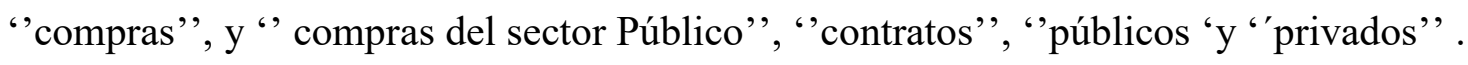
Las investigaciones seleccionadas se caracterizaron por ser en su mayoría de lengua inglés, portugués y español, además, aquellos que poseían relación con la variable de estudio o que de alguna manera poseían concordancia con los resultados que se querían obtener. En el buscador Scielo se encontraron artículos que pertenecían al idioma extranjero (inglés) y al español y poseían los requisitos requeridos, siendo un total de 28 del mismo modo se recolectaron 4 de Scopus, 1 de Ciencia Latina, los demás se obtuvieron de la base de datos de Scielo 14, Google Académico 2 y repositorios 
nacionales internacionales como el de la Universidad de Guadalajara, UPC, Universidad de Santiago de Compostela 3.

Los artículos para ser incluidos tuvieron que ser parte de una selección rigurosa, artículos pertenecientes a revistas indexadas conocidas y confiables, aquellos que tuvieran objetivos similares a los de esta investigación, resultados que contribuyan y fueran relevantes. Además, los artículos que tuvieran una variedad de autores, de modo que otorguen perspectivas diferentes y contribuyan para dar un panorama claro y real.

Por otro lado, los artículos que fueron descartados para su utilización en esta investigación fueron aquellos que no tenían las palabras clave adecuada, que no se hallaran completos, es decir, que no tuvieran conclusiones sin resultados, y mucho menos aquellos que no tuvieran bibliografía veraz, que no se relacionaran con la investigación o no aportaran de manera significativa al estudio. Asimismo, aquellos que no fueran parte del periodo de tiempo establecido o del idioma requerido.

Esta selección se llevó a cabo en tres filtros: el primer filtro se dio a selección de aquellos artículos que formaran parte de los buscadores requeridos, el segundo filtro fue la lectura de los títulos y de los resúmenes presentes, en el tercer filtro fue la lectura rigurosa de la parte de metodología, resultados y conclusiones.

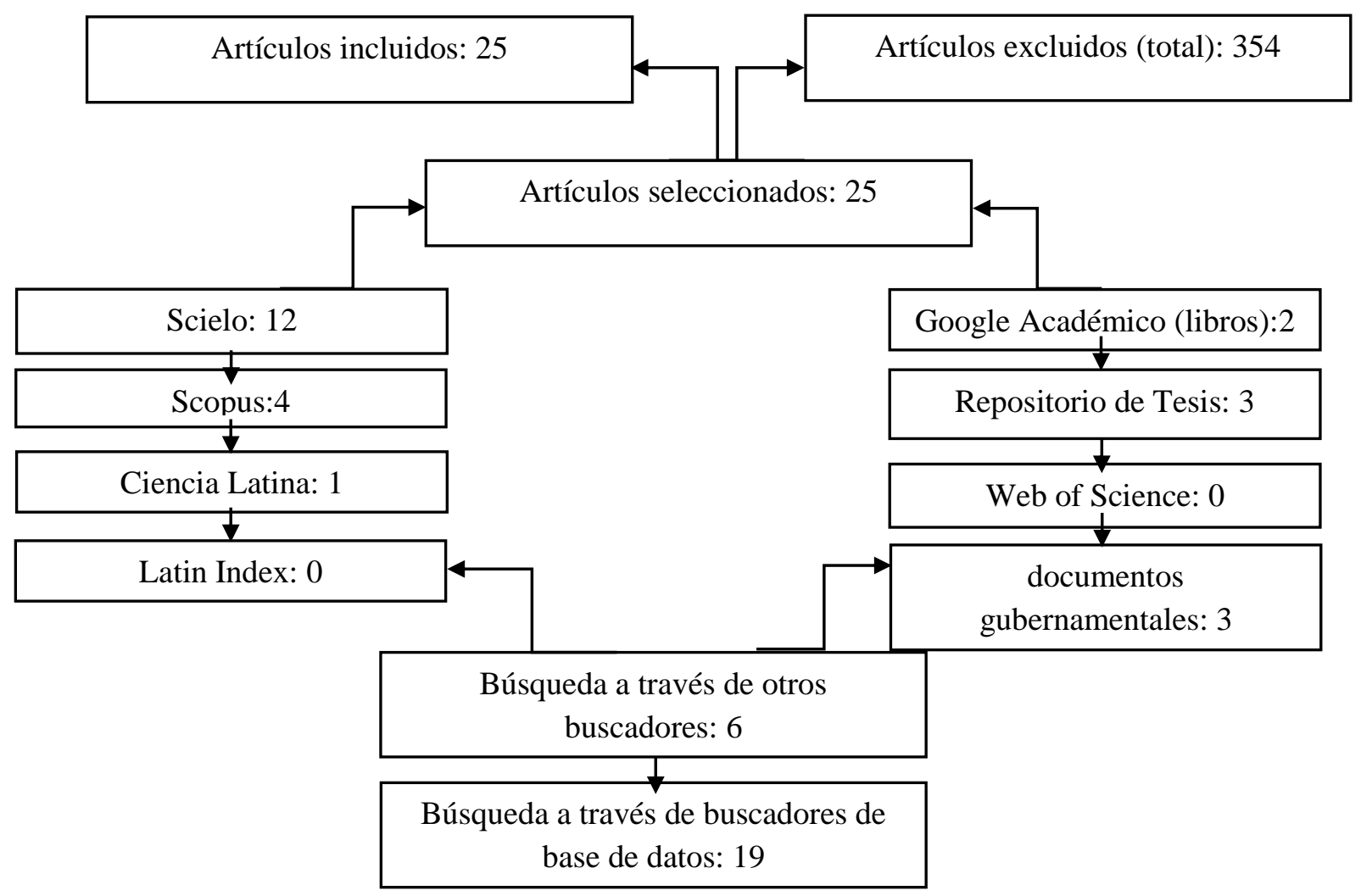

Figura: Esquema PRISMA 


\section{RESULTADOS Y DISCUSIÓN}

\section{Resultados}

En base a toda la recolección de datos y artículos seleccionados se pudo encontrar que las contrataciones en el sector público carecen muchas veces de trasparencia y rigor en la rendición de cuentas, es por esto que se convierte en un facilitador de delitos. Además, las autoridades cumplen un rol fundamental porque si ellos poseen una convicción débil en valores y una falta de compromiso con su deber de funcionario público estarían dando pie a que estos delitos afloren.

El sector público se encuentra sesgado por la búsqueda de fines personales, por tal motivo ha dejado de llevarse a cabo una inversión responsable que realmente sustente el gasto público y las atribuciones que la sociedad da y desea recibir. En líneas mayores, la administración que el Estado brinde debe ser en base a la eficacia y eficiencia del uso de recursos y su debida gestión, por ejemplo, lo organismos se encuentran obligados a realizar reparaciones o construir nuevos cimientos de infraestructura en caso los que se tengan no fueran suficiente o estos ya hayan cumplido su tiempo de vida, tales como instalaciones de alumbrado, aeropuertos, oficinas, entre otras.

Las contrataciones en el sector público se definen como los procesos en los cuales se identifican las necesidades de un determinado lugar para luego entregar el bien correcto en el tiempo adecuado y aun precio posible, lo cual se lleva acabo bajo un proceso trasparente y cumpliendo la ley que los resguarda, sin embargo, de la teoría a la practica existen problemas. Estas dificultades sean dan porque estas contrataciones requieren de tramites que toman tiempo y resultan ser tediosos, ya sea la inscripción en los padrones legales, contratos con intermediarios oficiales, la búsqueda del beneficio social, entre otros lineamientos, los cuales son evadidos muchas veces por personas inescrupulosas que intentan 'sacarle la vuelta a la ley'.

Por otro lado, los resultados también arrojaron que mientras mayor sea el Estado en cuanto a la cantidad de funcionarios y al tamaño de la nación mayor es la incidencia de actividades relacionadas a la corrupción, asimismo, se destinan grandes cifras de dinero a realizar compras públicas o adquirir servicios que no son necesarios o que muchas veces ya han sido requeridos anteriormente. Esto ocasionada que el Sector Público quede como un comprador que necesita mejorar los niveles de eficiencia, y ser más inteligente al realizar las compras, así como negociar mejores estrategias. Otro resultado pertinente es 
que el personal sea capacitado en realizar compras de manera adecuada, de modo que se desabollone habilidades para que se busque la innovación del país y a su vez el desarrollo de la sociedad en su conjunto. De esta manera obtener mejores compras y que los contratos se lleven a cabo de forma rápida y responsable.

Los autores consultados también indicaron que en las compras públicas se reporta gran cantidad de fraude, corrupción y complejidad en los procesos, un estudio de la Oficina de las Naciones Unidas contra la Droga y el Delito (OECD) (2020) indicó que el 57\% de países presentaron casos de corrupción relacionados a las compras, ya sea en casos de soborno, malversaciones, abuso de las mismas autoridades, mala rendición de cuentas, otros. Si bien debe llevarse una gestión pública que garantice y justifique el gasto público, ya sea en el fomento de producción de bienes, involucramiento de micro empresas, promover el cumplimiento de contratos, y que los suministros o servicios lleguen en óptimas condiciones.

En el caso del Perú, las contrataciones públicas son un instrumento sumamente importante para que el gobierno cumpla sus objetivos y beneficie a la sociedad en el transcurso, es por esto que, si un Estado no cumple con una administración de calidad y que priorice las necesidades sociales, automáticamente presentará inconformidad por parte de la población. Cabe señalar que las compras públicas deben ser sostenibles e innovadoras, lo cual muchas veces se ve resquebrajado por la falta de una visión estratégica que vaya más allá de gastar dinero y realizar obras porque se puede y no porque se necesite. Finalmente, existe una falta de control y supervisión en las compras estatales, así como una falta de estandarización, exigencia, mercado poco competitivo, y una capacidad de gestión en los recursos, lo cual es propio de los países desarrollados y en vías de desarrollo.

\section{Discusión}

En función del objetivo planteado en esta investigación, destacar la influencia de las contrataciones del Estado en el desarrollo del país, se expondrán 8 artículos que poseen características e información pertinente para ser contrastado y tomado en cuenta a continuación, asimismo, contribuye significativamente a este artículo de revisión.

Jun \& Joong (2018) entre sus resultados, ha habido un incremento en las obras públicas de gran escala, sobre todo las asociaciones entre público-privadas, las cuales generalmente se desvían de los lineamientos. Por tal motivo, es necesario que se 
establezcan medidas de anticorrupción para promover la responsabilidad por parte de los funcionarios y organismos gubernamentales, ya que esta herramienta contribuye al desarrollo del país si se realizan todos los pasos para realizar las compras y si cumplen con las características necesarias, tales como el beneficio social, un precio justo, condiciones óptimas y contratos trasparentes e individuales. El propósito de las compras del sector público debe ser privilegiar el interés general y minimizar la desigualdad, inclusión e innovar, pero muchas de ellas no cumplen ninguno de los principios ya mencionados.

Por su parte, Chiappinelli (2020) indicó que, las autoridades "rellenan" costos y se involucran en actos de corrupción porque buscan el beneficio propio en las obras o servicios que se contraten, lo cual influye negativamente en el Estado, ya que debido a ellos la sociedad tienen una desconfianza hacia la política. Los casos de fraude y corrupción en la contratación estatal siguen aumentando y las autoridades no se dan abasto para controlar la situación, además, las penas establecidas para estos actos no son suficientes y muchas veces la impunidad se hace presente. Los contratos que se firmen deben garantizar un interés público, sin embargo, el abuso del cargo y el poder se ha considerado como uno de los problemas más graves actualmente. Pese a que existe controles y medidas de anticorrupción para los asuntos de compras, como la promoción de las buenas prácticas, integridad, ética, colaboración ciudadana y supervisión, esto sigue siendo ineficaz. Por tal motivo el autor concluye que deben plantearse sanciones ejemplares para prevenir estos delitos en las contrataciones del sector público, siendo sanciones monetarias, inhabilitación del cargo, privación de la libertad, otros.

Los autores concluyeron que las compras en el sector público se encuentran plagadas de delitos relacionados al abuso del poder, ventajas políticas y la búsqueda de beneficios de grupos cerrados. Por otro lado, las prácticas llevabas a cabo con una contratación electronóca ha mejorado los procesos, primando los objetivos como la promoción de bienes y obras en favor del país, y el interés de la sociedad, todo esto de forma eficiente y responsable. Otro punto importante a destacar es que las compras públicas deben ser supervisadas y controladas para sustentar al ciudadano el gasto que se viene haciendo, es decir, debe ser una herramienta aliada y no como un ente sancionador que busca irregularidades en los procesos (Rodrigues \& Gomes, 2021). 
El Instituto Nacional de Administración Pública (2019) en su análisis fijó que los gobiernos tienen la obligación de mejorar la eficiencia y eficacia en las contrataciones públicas, ya que estas influyen positivamente en el crecimiento, desarrollo y gestión del país. Es por esto que deben revisar y actualizar las obras que se estén realizando, de modo que formen parte del conocimiento de la sociedad, así como capacitar al personal, innovar y acelerar los procesos para que funcionen acorde a la programación y evaluación. Sin embargo, lo más importante es que el Estado genere una política de trasparencia fundamentada en valores como la ética y la honestidad.

Lemke \& Werner (2020) obtuvieron que, desde el año 2015 ha disminuido el rendimiento de cuentas por parte de los Estados, y ahora, debido a la pandemia por la COVID-19, las compras de elementos de bioseguridad y equipamiento para el área de salud se ha disparado, al igual que el presupuesto. Tal es el caso de la UE, uno de los países que realizó grandes compras en recursos como mascarillas, botellas de alcohol, camas UCI y otros recursos, pese a haber pagado un total de 6.7 millones de euros a la empresa a cargo, estos instrumentos jamás llegaron al destino. Cabe señalar que este no ha sido el único caso registrado, pero si uno de los pocos que ha sido descubierto. Por otro lado, existe una falta de control interno, lo cual repercute en información desactualizada del presupuesto y falta de documentos oficiales que sirvan para contabilizar las obras, servicios y a su vez, los estados financieros, retrayendo el desarrollo del país.

Ortega (2021) determinó que, el uso de plataformas virtuales para registrar las compras públicas cada vez se hace más popular y necesario porque permite que la población pueda acceder y supervisar el gasto público y las obras que se vienen realizando para su beneficio. Además, los gobiernos vienen realizando compras que apuestan por la innovación y el desarrollo, los cuales se ven reflejados en proyectos de gran escala en los distintos ámbitos, pero la supervisión y evaluación debe ser mas rigurosa, de modo que se eviten la tergiversación del dinero.

Caetano et al. (2020) indicó que, los países han incrementado su nivel de compras basado en la modernización del sistema, siendo este un proceso trasparente y de buenas prácticas, sin embargo, no es el caso de todos los Estados. A su vez, los funcionarios públicos desempeñan un papel importante porque como actores responsables no pueden escudarse en mala praxis y la trasgresión de las leyes, pese a que existen autoridades y proveedores que se relacionan para incrementar su "sueldo" a base de "regalitos". Las compras 
estatales son una herramienta para el desarrollo y corrección de problemas que aquejan a la ciudadanía (Soto \& Quiñones, 2017).

Finalmente, Martins \& Sano (2018) señaló que, la contratación pública es un proceso que es complejo y se da de manera lenta, debido a que converge la relación entre las necesidades y los proveedores, los cuales poseen un cronograma, por ejemplo, toda compra inicia con una necesidad que es comunicada de la sociedad al Estado, luego se procede a establecer los costos y el proceso, seguidamente la selección del mejor proveedor (elección en base al precio y la calidad del bien y servicio), el cierre del contrato y el establecimiento de la fecha de entrega. Todos estos pasos, llevándonos acordemente y de forma responsable influyen positivamente en el crecimiento del país, sin embargo, debido a que las autoridades esquivan procesos y no existe una correcta supervisión, las compras no gozan de una planificación ni de un proceso de compra exitosa.

\section{CONCLUSIÓN O CONSIDERACIONES FINALES}

- Como primera conclusión, se obtuvo que las contrataciones del Estado favorecen en gran medida al desarrollo del país por la inversión que estas representan y por el alto consumo estatal que producen en el mercado interno.

- Como segunda conclusión, la participación de las empresas privadas dentro de las contrataciones del Estado, resultan ser de gran importancia, sin embargo, son muy pocas las que se basan en la transparencia, calidad e integridad. Las empresas proveedores tienen la obligación de exigir contratos adecuados que no estén orientados a lucrar, sino que se contribuya al bienestar de la sociedad.

- Como tercera conclusión, existe una fuerte corrupción representada en obras y contrataciones que el Estado realiza, los cuales normalmente tienen implicancia con empresas privadas, por ejemplo, elevando precios en las obras públicas, acuerdos fantasmas, desvió de fondos, entre otras acciones.

- Como cuarta conclusión, la implementación de controles de inventario tales como supervisión del personal, filtros para los recursos adquiridos y seguimiento al proceso de compra deben ser producto de una importante gestión pública que el Estado tiene la obligación de realizar, sin embargo, en la realidad esto no se ejecuta de la manera adecuada. 
- Como última conclusión, la condicionante en los aspectos de obligaciones sociales y medio ambientales deben ser priorizantes a la hora de firmar contrataciones que no afecten el desarrollo de ambos, al contrario que fomenten su crecimiento, de tal manera que se aprovechen los recursos disponibles.

\section{LISTA DE REFERENCIAS}

Naciones Unidas contra la Droga y el delito. (2020). Corruption in public procurement.

UNODC. Obtenido de https://www.unodc.org/e4j/es/anti-corruption/module4/key-issues/corruption-in-public-procurement.html

Anibal, A. M., \& Sano, H. (2018). Purchasing function in the public sector: challenges to promote agility in electronic reverse auctions. Scielo. Obtenido de https://www.scielo.br/j/rap/a/YQ35TgSsFSq3J8RvVpNMjpB/abstract/?lang=en

Bonelli, F., \& Cabral, S. (2018). Effects of Capabilities on the Performance of Public Sector Service Contracts. Scielo. Obtenido de https://doi.org/10.1590/19827849rac2018170152

Caetano, R., Almeida, P., Villela, M., Villardi, P., \& Serpa, C. (2020). The case of eculizumab: litigation and purchases by the Brazilian Ministry of Health. Revista de Saúde Pública, 54(22), 1-11. doi:https://doi.org/10.11606/s15188787.2020054001693

Chiappinelli, O. (2020). Political corruption in the execution of public contracts. Journal of Economic Behavior \& Organization, 179, 116-140. doi:https://doi.org/10.1016/j.jebo.2020.08.044

Espinoza, E. (2018). Variables and their operationalization in educational research. Part I. Conrado, 39-49. $14(1), \quad$ Obtenido de http://scielo.sld.cu/scielo.php?script=sci_arttext\&pid=S199086442018000500039

Espitia, J., Ferrari, C., Gonzales, J., Reyes, L., Romero, A., Tassara, C., . . . Zafra, G. (2019). Public spending in Colombia. Reflections and proposals. Scielo. Obtenido de http://www.scielo.org.co/scielo.php?script=sci_arttext\&pid=S012459962019000100291

Francklin, R., Aspirino, M., Sarache, J., \& Leon, F. (2019). Intelligent System for the Contracts Automatic Generation within the framework of the Public Procurement Law. Scielo. Obtenido de 
http://scielo.senescyt.gob.ec/scielo.php?script=sci_arttext\&pid=S139001292019000400041

Gerrero, M. M., \& Gimeno, J. F. (2020). Observatorio de los contratos públicos. Aranzadi. Obtenido de https://books.google.es/books?hl=es\&lr=\&id=EBCEAAAQBAJ\&oi=fnd\&pg=PT5\&dq=contratos $+\mathrm{p} \% \mathrm{C} 3 \%$ BAblicos $+\mathrm{y}+\mathrm{priva}$ dos\&ots=05xt9mz4Tg\&sig=kUWAoyvZ6hHNfirK7KnHiIJVtY\#v=onepage \&q\&f=false

Gonzales, M. B. (2017). What is purchased, how and from whom by Seguro Popular in Mexico? Experience with strategic purchasing at national level and in a pioneer institution.

Scielo.

Obtenido

de https://www.scielosp.org/article/spm/2017.v59n1/59-67/

Instituto Nacional de Administración Pública. (2019). Importancia económica de las contrataciones del sector público: propuestas del nuevo gobierno para mejorar su eficiencia y eficacia económica. Revista de Administración Pública, 54(2), 1-24.

Jung, Y., \& Joong, M. (2018). Contracting-out public-private partnerships in mega-scale developments: The case of New Songdo City in Korea. Cities, 72(4), 43-50. doi:https://doi.org/10.1016/j.cities.2017.07.021

Karunananthan, S. (2021). When is systematic review replication useful and when is it wasteful? Letter to the editor, 45. doi:https://doi.org/10.26633/RPSP.2021.11

Lemke, W., \& Werner, T. (2020). Dissecting long-term Bund yields in the run-up to the ECB's public sector purchase programme. Journal of Banking \& Finance, 111. doi:https://doi.org/10.1016/j.jbankfin.2019.105682

Martins, A., \& Sano, H. (2018). Purchasing function in the public sector: challenges to promote agility in electronic reverse auctions. Brazilian Journal of Public Administration, 58(1), 1-18. doi:https://doi.org/10.1590/0034-7612164213

Ortega, C. (2021). The State as a smart consumer to carry out public procurement of innovation. PAAKAT: revista de tecnología y sociedad, 11(20). doi:https://doi.org/10.32870/pk.a11n20.532

Payne, E. I. (2017). The state, the elite and the communities: The contracts around the exploitation of the madreperla in the pacific of Costa Rica (XIX and XX centuries). Scielo. Obtenido de https://www.scielo.sa.cr/scielo.php?pid=S221541752017000100225\&script=sci_arttext 
Peñate, M. V. (2020). La compra publica de innovaciones como politica de demanda en sectores estrategicos. Analisis de la experiencia española desde la perspectiva regional. Tesis doctoral, Universidad de Santiago de Compostola. Obtenido de http://hdl.handle.net/10347/26053

Pinto, R. M., Paiva, E. d., Pinto, R. M., \& Oliveira, E. d. (2018). Contract inspecting of outsourced service: challenges for public universities. Scielo. Obtenido de https://www.scielo.br/j/gp/a/L6vZC4dSZL8py78dGBm4xrz/?lang=en

Ramió, C. (2020). Inteligencia artificial y administracion publica: Robots y humanos compartiendo el servicio publico. Catarata. Obtenido de https://books.google.es/books?hl=es\&lr=\&id=rpOEDwAAQBAJ\&oi=fnd\&pg= PT6\&dq $=$ compra + p\%C3\%BAblica + sector + p $\%$ C3\%BAblico\&ots $=$ X2CCsvI4sP \&sig=kjzWVUMZr6mlzqa8v_rHrEQ6II\#v=onepage \&q=compra\%20p\%C3\%BAblica\%20sector\%20p $\% \mathrm{C} 3 \%$ BAblico\&f $=$ false

Rodrigues, M., \& Gomes, J. (2021). Does the size of the government increase corruption? An analysis for Brazilian municipalities. Brazilian Journal of Public Administration, 55(2), 272-291. doi:https://doi.org/10.1590/0034-761220200690 Rodriguez, M. (2020). The public and the private healthcare sector in Spain: stability or change? Scielo Obtenido de https://www.scielosp.org/article/gs/2019.v33n6/499-501/es/

Ruiz, S. C., \& Delgado, J. B. (2020). El control interno en el proceso de contrataciones en las instituciones públicas. Ciencia Latina. doi:https://doi.org/10.37811/cl_rcm.v4i2.159

Soto, R., \& Quiñones, V. (2017). Study of state acquisitions and technologies of nformation: the case of the electronic procurement system of the State in Peru . Science and Technology, 12(1), 1-16. 